\title{
Bedeutung regional-ökonomischer Faktoren für die Entscheidung von Eigenheimbesitzern
}

Der Gebäudezustand in zwei brandenburgischen Regionen unterscheidet sich trotz unterschiedlicher Wachstumsdynamik und Nutzungsperspektiven kaum. Für die Sanierungsentscheidung spielen regionalökonomische Faktoren bei selbstnutzenden Eigentümer/innen gegenüber individuellen Motiven nur eine untergeordnete Rolle. Von Julika Weiß, Elisa Dunkelberg, Maike Gossen und Doreen Großmann

\section{Einführung}

Mit dem Energiekonzept hat die Bundesregierung im Jahr 2010 eine langfristige Strategie zur zukünftigen Ausrichtung der Energieversorgung und der Klimaschutzziele in Deutschland festgelegt. Neben der Gewährleistung der Versorgungssicherheit und der Wirtschaftlichkeit soll die Energieversorgung umweltverträglich werden. Für die Raumwärmeerzeugung wurden in Deutschland im Jahr 2013753 Milliarden Kilowattstunden (kWh) benötigt, dies entspricht einem Anteil von $29 \%$ des Endenergieverbrauchs (BMWi 2014a). Der Bereich der Raumwärme hat somit einen wichtigen Stellenwert bei der Umsetzung des Energiekonzeptes und der Erreichung der Klimaschutzziele. Daher legte die Bundesregierung in ihrem Energiekonzept fest, dass der Gebäudebestand bis zum Jahr 2050 nahezu klimaneutral werden soll (BMWi/BMU 2010). Dies bedeutet, dass einerseits die Effizienz der Gebäude durch energetische Sanierungsmaßnahmen erhöht werden soll, um dadurch eine deutliche Reduzierung des Endenergieverbrauchs zu erreichen. Anderseits soll der verbleibende Endenergieverbrauch vorzugsweise durch Erneuerbare Energien gedeckt werden.

Mit Abstand den größten Anteil am Endenergieverbrauch für die Raumwärmeerzeugung entfällt auf die privaten Haushalte (66\% im Jahr 2013) (BMWi 2014a). Auf den Anwendungsbereich Gewerbe, Handel und Dienstleistungen beziehungsweise Industrie entfielen im Jahr 2013 lediglich $25 \%$ beziehungsweise 9\% der Raumwärme (BMWi 2014a). Somit sind die privaten Haushalte ein wichtiger Akteur bei der Erreichung der Klimaschutzziele des Energiekonzepts der Bundesregierung.
Bundesweit sind $82 \%$ der Wohngebäude Ein- und Zweifamilienhäuser (EZFH) (Diefenbach et al. 2010); in Brandenburg liegt ihr Anteil sogar bei $86 \%$ (AStBB 2011). Diese werden zu $95 \%$ von selbstnutzenden Eigentümer/innen bewohnt (Zensus 2011). Während im Neubau bereits durch die geltende Energieeinsparverordnung eine hohe Gebäudeeffizienz verpflichtend ist und zukünftig sogenannte Nullemissionshäuser Standard sein werden, stellt der Gebäudebestand das zentrale Problemfeld dar. Der Hauptanteil des bundesweiten Wohngebäudebestandes (64\%) wurde bis zum Jahr 1978 (Zensus 2011) errichtet - erst im Jahr 1977 trat die erste Wärmeschutzverordnung in den alten Bundesländern in Kraft. Durch die vorhandene Bausubstanz weist dieser Altbaubestand eine geringe Energieeffizienz hinsichtlich der Raumwärme auf. Bei lediglich 25 bis $30 \%$ wurde die Effizienz durch eine energetische Sanierung der Gebäudehülle erhöht (Diefenbach et al. 2010). Zugleich sind die Hemmnisse bei einer energetischen Sanierung hoch; beispielsweise spielen mangelndes Problembewusstsein, fehlende Bereitschaft zur Finanzierung der erforderlichen Kosten, ausbleibende systematische Instandhaltungsplanung sowie Ängste vor Dreck, Stress und Bauschäden für die Eigentümer/ innen eine wichtige Rolle (Stieß et al. 2010). Zudem weist bei derzeitigen Sanierungsstandards rund jedes sechste Gebäude bauliche Sanierungshemmnisse auf (Weiß/Dunkelberg 2010; Loga et al. 2007). Restriktionen existieren insbesondere für die Dämmung der Außenwände (z. B. Sichtmauerwerk, Denkmalschutz, Stuck/Ornamente, Erscheinungsbild), aber auch für die Dämmung der obersten Geschossdecke (z. B. nicht zugänglich) und Kellerdecke (z. B. Installationen unter der Decke) (Jochum et al. 2012). Die Bedeutung dieser Hemmnisse kann bei höheren Sanierungsanforderungen noch steigen.

Eine klimafreundliche Wärmeversorgung kann insbesondere durch den Einsatz Erneuerbarer Energien sowie KraftWärme-Kopplungs-Lösungen sichergestellt werden. Allerdings sind hier die bisherigen Ausbauzahlen gering: So lag der Anteil von Wärme aus Erneuerbaren Energien im Jahr 2013 bei nur 9,1\% und stagnierte in den letzten Jahren (BMWi 2014b).

Sowohl die Sanierung der Gebäudehülle als auch der Einsatz Erneuerbarer Energien sind häufig hochinvestive Maßnahmen. Die Finanzierung durch Eigentümer/innen und andere Investoren kann durch kurze Nutzungsperspektiven gehemmt werden. Eigentümer/innen, die absehen können, dass sie nach kurzer Zeit wieder umziehen, haben ebenso wenig 
ein Interesse an langfristigen Investitionen wie solche, die damit rechnen müssen, dass sie keine Nachnutzer/innen oder Käufer/innen für ihre Immobilie finden werden. Das Projekt "Gebäude-Energiewende" geht von der These aus, dass die Nutzungsperspektiven zu einem ganz erheblichen Teil durch äußere Rahmenbedingungen wie die regionalökonomische Situation geprägt werden [1]. Dadurch wird ein spürbarer Unterschied zwischen energetischen Sanierungsmustern in Entleerungsräumen und Wachstumsregionen erwartet. Die Nutzungsperspektive hat gleichzeitig einen Einfluss darauf, welche Sanierungslösungen aus ökologischer und ökonomischer Sicht vorteilhaft sind, denn Sanierungen mit hohem Ressourcen- und Investitionsbedarf lohnen sich nur langfristig.

Der vorliegende Beitrag geht der Frage nach, inwiefern die regionalökonomische Situation als äußere Rahmenbedingung eine relevante Einflussgröße für das Sanierungsgeschehen und die individuelle Sanierungsentscheidung darstellt. Hierfür werden zunächst zwei Regionen in Brandenburg mit unterschiedlicher Wachstumsdynamik vorgestellt und zentrale Parameter zur regionalökonomischen Situation vergleichend ausgewertet. In einem zweiten Schritt wird der Bestand an EZFH in beiden Regionen verglichen und die Quantität sowie die Tiefe der energetischen Sanierungsaktivität der letzten Jahre ausgewertet. Anschließend wird die individuelle Sanierungsentscheidung beleuchtet und der Einfluss der regionalökonomischen Situation auf die individuelle Sanierungsentscheidung analysiert; Grundlage bildet hier eine qualitative Befragung von Hauseigentümer/innen. Basierend auf diesen drei empirischen Untersuchungen werden abschließend Schlussfolgerungen zur Bedeutung regionalökonomischer Faktoren gezogen.

\section{Charakterisierung der Untersuchungs- regionen}

Als Untersuchungsregionen wurden zwei Gebiete in Brandenburg gewählt, die zwar räumlich nahe beieinander liegen, jedoch ganz unterschiedliche Wachstumsdynamiken aufweisen: die Stadt Potsdam und der Landkreis Potsdam-Mittelmark sind eine Wachstumsregion, während die Planungsregion Lausitz-Spreewald mit den Landkreisen Dahme-Spreewald, ElbeElster, Oberspreewald-Lausitz, Spree-Neiße sowie der kreisfreien Stadt Cottbus eine überwiegend schrumpfende Region darstellt.

Die Einwohnerzahl in der Region Lausitz-Spreewald ist von 1990 bis 2011 um 17\% zurückgegangen; in der Region Potsdam/Potsdam-Mittelmark ist sie hingegen um die gleiche Größenordnung angestiegen (AStBB 2012b). Ursachen für die überdurchschnittliche Bevölkerungsschrumpfung in der Region Lausitz-Spreewald sind der hohe Wegzug und die niedrige Geburtenrate. In Potsdam/Potsdam-Mittelmark ist ein $\mathrm{Zu}$ zug zu erkennen und auch die Geburtenrate liegt hier deutlich höher. Für die nächsten Jahre wird prognostiziert, dass dieser Trend in beiden Regionen anhält (AStBB 2012 b). Für Lausitz-
Spreewald wird bis zum Jahr 2030 ein weiterer Bevölkerungsrückgang von etwa $15 \%$ erwartet, in Potsdam/Potsdam-Mittelmark hingegen eine weitere Bevölkerungszunahme von etwa $5 \%$. Die Bevölkerungsdichte ist dabei in Lausitz-Spreewald mit 86 Einwohner/innen je Quadratkilometer bereits heute deutlich geringer als in Potsdam/Potsdam-Mittelmark mit 132 Einwohner/innen je Quadratkilometer (AStB B 2012 b).

Deutliche Unterschiede bestehen auch bezüglich Einkommen und Durchschnittsalter. So lag 2011 das durchschnittliche monatliche Netto-Haushaltseinkommen in Lausitz-Spreewald rund 350 Euro unter dem in Potsdam/Potsdam-Mittelmark. Allerdings haben Haushalte in Deutschland monatlich durchschnittlich rund 1.000 Euro mehr zur Verfügung als die in Potsdam/Potsdam-Mittelmark - bei annährend identischer Personenanzahl je Haushalt (DESTATIS 2012, AStBB 2012a). In beiden Regionen steht den Einwohner/innen also weniger Geld zur Verfügung als im bundesweiten Durchschnitt. Den Unterschieden im Haushaltseinkommen entsprechend war der Anteil an Renten- und Sozialleistungsempfänger/innen in Lausitz-Spreewald im Jahr 2011 mit 43\% deutlich höher als in Potsdam/Potsdam-Mittelmark mit 30\% (AStB B 2012 c). Der Anteil erwerbstätiger Personen ist hingegen in Potsdam/Potsdam-Mittelmark höher und die Einwohner/innen sind durchschnittlich drei Jahre jünger als in Lausitz-Spreewald (AStBB 2012b).

\section{Gebäudebestand und energetischer Zustand der Gebäude}

Die vorgestellten Zahlen zeigen, dass sich die beiden untersuchten Regionen hinsichtlich ihrer regionalökonomischen Situation deutlich voneinander unterscheiden. Nachfolgend wird nun der Frage nachgegangen, ob sich die regionalen Unterschiede im energetischen Zustand der Wohngebäude widerspiegeln. Im Fokus stehen Wohngebäude im Eigentum von Privatpersonen und EZFH.

Insgesamt gibt es in Potsdam/Potsdam-Mittelmark etwa 81.000 Wohngebäude, von denen $84 \%$ EZFH sind. Mit fast 160.000 Wohngebäuden ist der Wohngebäudebestand in Lausitz-Spreewald fast doppelt so groß bei ähnlich hohem Anteil an EZFH. Die EZFH sind in den Untersuchungsregionen zu über $95 \%$ in Privatbesitz. Ein Großteil der EZFH wurde in beiden Regionen vor 1948 erbaut (siehe Abbildung 1). In den Jahren der DDR (1949-1990) war der Zubau im Gegensatz zu den alten Bundesländern gering. Damit weisen die beiden Untersuchungsregionen einen höheren Anteil an älteren EZFH, die vor 1948 erbaut wurden, auf als im Bundesdurchschnitt.

Die folgenden Auswertungen zum energetischen Zustand der Gebäude in den Untersuchungsregionen basieren auf einem Datensatz der co2online $\mathrm{GmbH}$, der auf freiwilligen Eingaben in interaktiven Online-Beratungsinstrumenten beruht. Der Datensatz ist nicht repräsentativ für die Wohngebäude in den Untersuchungsregionen, aber angesichts bundesweiter hoher Übereinstimmungen mit anderen Datensätzen (Jahnke 
2013) wird davon ausgegangen, dass dieser insgesamt gut mit den Eigenschaften der Gesamtheit der Wohngebäude übereinstimmt. Für Lausitz-Spreewald basiert die Auswertung auf Daten von 2.931 Gebäuden und in Potsdam/Potsdam-Mittelmark von 2.426 Gebäuden - wobei jeweils nur bei einem Teil Informationen zu den durchgeführten Sanierungsmaßnahmen vorlagen (Dunkelberg/Weiß 2015).

Die Abbildung 2 zeigt, dass erstens die bis 1990 erbauten Gebäude in allen Baualtersklassen einen ähnlich hohen mittleren Heizenergieverbrauch aufweisen und erst die nach 1990 erbauten Gebäude deutlich geringere Heizenergieverbräuche haben und dass zweitens die Unterschiede zwischen den Untersuchungsregionen sehr gering sind. Im Durchschnitt liegt der Heizenergieverbrauch in Lausitz-Spreewald bei $143 \mathrm{kWh}$ pro Quadratmeter und Jahr $\left(\mathrm{m}^{2} \mathrm{a}\right)$, in Potsdam/Potsdam-Mittelmark bei $136 \mathrm{kWh} /\left(\mathrm{m}^{2} \mathrm{a}\right)$ und bundesweit bei $144 \mathrm{kWh} /\left(\mathrm{m}^{2} \mathrm{a}\right)$.

Aus dem Datensatz der co2online GmbH wurden durchschnittliche jährliche Sanierungsraten für Fünf-Jahres-Zeiträume gebildet (siehe Tabelle 1). Insbesondere die Sanierungsraten für die Maßnahmen Fenster- und Heizungserneuerung waren in den Untersuchungsregionen zwischen 1990 und 2009 im Vergleich zu Gesamtdeutschland hoch. Eine Betrachtung der Einzelperioden zeigt jedoch, dass Heizungen primär in den 1990er Jahren ausgetauscht wurden. Die Sanierungsraten zwischen 2000 und 2009 sind hier deutlich geringer als im bundesweiten Durchschnitt. Auch Fassadendämmungen wurden in den 1990er Jahren in den Untersuchungsregionen häufiger umgesetzt als im bundesweiten Durchschnitt. Zwischen den beiden Untersuchungsregionen sind die Unterschiede dagegen nicht so deutlich. Tendenziell waren Sanierungen in Lausitz-Spreewald in den 1990ern häufiger und in Potsdam/Potsdam-Mittelmark ab dem Jahr 2000.

Um den Sanierungszustand näher zu beleuchten, wurden im nächsten Schritt verschiedene energetische Sanierungsmaßnahmen an der Gebäudehülle gemeinsam ausgewertet. Im Vergleich zu Deutschland ist der Anteil vollsanierter EZFH in beiden Regionen größer (siehe Tabelle 2), der Anteil unsanierter EZFH ist im Vergleich zu Deutschland vor allem in der Baualtersklasse 1949 bis 1990 deutlich geringer.

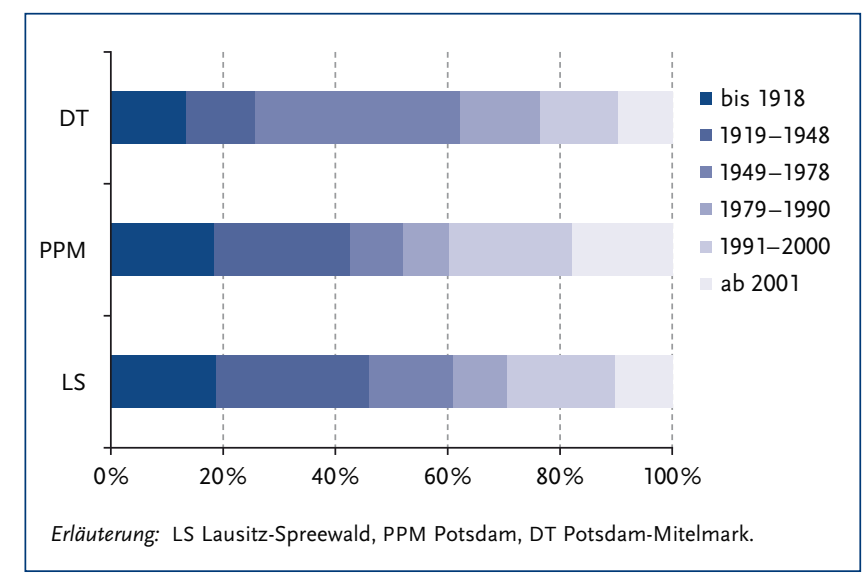

Abbildung 1: Prozentuale Verteilung der EZFH in Baualtersklassen (AStBB 2014; DESTATIS 2014)

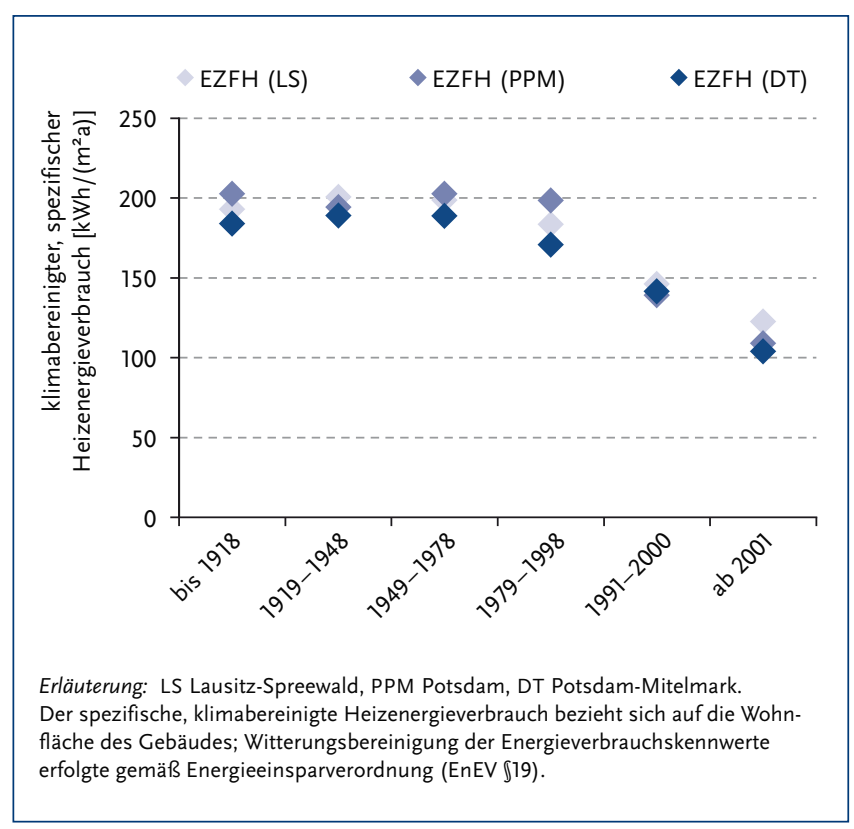

Abbildung 2: Durchschnittlicher spezifischer, klimabereinigter Heizenergieverbrauch von Ein- und Zweifamilienhäusern

(eigene Auswertung eines Datensatzes der co2online GmbH von 2014)

\begin{tabular}{|c|c|c|c|c|c|c|}
\hline Bauteil & Region & 1990-1994 & 1995-1999 & $2000-2004$ & 2005-2009 & 1990-2009 \\
\hline \multirow[t]{3}{*}{ Fenster } & LS & $2,3 \%$ & $5,1 \%$ & $2,7 \%$ & $2,2 \%$ & $3,1 \%$ \\
\hline & PPM & $2,3 \%$ & $3,6 \%$ & $3,2 \%$ & $1,1 \%$ & $2,6 \%$ \\
\hline & DT & $1,1 \%$ & $1,6 \%$ & $1,8 \%$ & $1,7 \%$ & $1,6 \%$ \\
\hline \multirow[t]{3}{*}{ Fassade } & LS & $1,0 \%$ & $2,0 \%$ & $0,9 \%$ & $0,4 \%$ & $1,1 \%$ \\
\hline & PPM & $0,7 \%$ & $1,3 \%$ & $1,5 \%$ & $0,9 \%$ & $1,1 \%$ \\
\hline & DT & $0,3 \%$ & $0,5 \%$ & $0,6 \%$ & $0,7 \%$ & $0,5 \%$ \\
\hline \multirow[t]{3}{*}{ Dach } & LS & $1,1 \%$ & $2,7 \%$ & $1,7 \%$ & $1,2 \%$ & $1,7 \%$ \\
\hline & PPM & $0,5 \%$ & $1,8 \%$ & $2,4 \%$ & $1,2 \%$ & $1,5 \%$ \\
\hline & DT & $0,8 \%$ & $1,0 \%$ & $1,4 \%$ & $1,2 \%$ & $1,1 \%$ \\
\hline \multirow[t]{3}{*}{ Heizung } & LS & $5,2 \%$ & $3,8 \%$ & $1,0 \%$ & $0,8 \%$ & $2,7 \%$ \\
\hline & PPM & $5,6 \%$ & $3,4 \%$ & $1,2 \%$ & $0,8 \%$ & $2,7 \%$ \\
\hline & $\overline{D T}$ & $2,4 \%$ & $2,3 \%$ & $2,5 \%$ & $1,8 \%$ & $2,3 \%$ \\
\hline
\end{tabular}

Tabelle 1: Jährliche Sanierungsraten der bis 1990 erbauten EZFH (eigene Auswertung eines Datensatzes der co2online GmbH von 2014) 
Da in Lausitz-Spreewald der Anteil sanierter EZFH über dem Sanierungsgrad in Potsdam/Potsdam-Mittelmark liegt, widersprechen die Zahlen der Vermutung, dass in wachsenden Regionen mehr energetisch saniert wird und wurde als in schrumpfenden Regionen. Ein möglicher Grund für den geringen Anteil teilsanierter EZFH in Potsdam/Potsdam-Mittelmark im Vergleich zu Lausitz-Spreewald kann die ohnehin gute Verkaufbarkeit von Gebäuden in wachsenden Regionen sein, die die Umsetzung von energetischen Sanierungen hemmt. Auch steht ein größerer Anteil der Wohngebäude in Potsdam/Potsdam-Mittelmark unter Denkmalschutz (13\% aller Wohngebäude in Potsdam Stadt versus 1\% der Wohngebäude in Lausitz-Spreewald (Online-Datenbank des Brandenburgischen Landesamtes für Denkmalpflege [DB1]).

Es fällt auf, dass die durchschnittlichen Heizenergieverbräuche in Lausitz-Spreewald und Potsdam/Potsdam-Mittelmark trotz des höheren Anteils vollsanierter Gebäude nicht geringer sind als im bundesweiten Durchschnitt. Dies deutet auf geringere Sanierungstiefen hin. Die mittleren Heizenergieverbräuche vollsanierter Gebäude bestätigen diese Annahme: In den Untersuchungsregionen liegen die Durchschnittswerte bei vollsanierten EZFH bei 134 und $150 \mathrm{kWh} /\left(\mathrm{m}^{2} \mathrm{a}\right)$. Im bundesweiten Durchschnitt hingegen ist der mittlere Heizenergieverbrauch vollsanierter EZFH mit $112 \mathrm{kWh} /\left(\mathrm{m}^{2} \mathrm{a}\right)$ deutlich niedriger.

Insgesamt zeigen die Daten, dass EZFH in den Nachwendejahren in den Untersuchungsregionen wie insgesamt in den ostdeutschen Bundesländern sehr viel saniert wurden - insbesondere Fenster und Heizungen wurden oftmals ausgetauscht. Der Einfluss regionalökonomischer Unterschiede und regionaler Wachstumsdynamiken wurde daher bislang vermutlich durch die Nachwendebesonderheiten - hoher Bedarf an und hohe Motivation zur Modernisierung in den 1990er Jahren überlagert. Gleichzeitig wirkt sich die Dynamik in den 1990er Jahren auch weiterhin auf das Sanierungsgeschehen aus. Die in den Nachwendejahren eingebauten Heizungskessel sind inzwischen in die Jahre gekommen, sodass in den nächsten Jahren eine hohe Austauschrate zu erwarten ist.

\begin{tabular}{lllll}
\hline \multicolumn{5}{c}{$\begin{array}{l}\text { Anteil der an der Gebäudehülle } \\
\text { sanierten EZFH }\end{array}$} \\
\cline { 2 - 5 } BA-Klasse & Region & unsaniert & teilsaniert & vollsaniert \\
\hline bis 1948 & LS & $11 \%$ & $67 \%$ & $22 \%$ \\
\cline { 2 - 5 } & PPM & $41 \%$ & $43 \%$ & $16 \%$ \\
\cline { 2 - 5 } & Dt & $30 \%$ & $66 \%$ & $3 \%$ \\
\hline $1949-1990$ & LS & $22 \%$ & $66 \%$ & $12 \%$ \\
\cline { 2 - 5 } & PPM & $20 \%$ & $58 \%$ & $22 \%$ \\
\cline { 2 - 5 } & Dt & $46 \%$ & $52 \%$ & $2 \%$ \\
\hline
\end{tabular}

Erläuterung: BA (Baualter); unsaniert: weder Kellerdecke, Fassade, Fenster, Dach noch oberste Geschossdecke wurden nach 1990 saniert; teilsaniert: mindestens eines der genannten Bauteile wurde nach 1990 saniert; vollsaniert: sowohl Fassade, Fenster und Dach oder oberste Geschossdecke wurden nach 1990 saniert.

Tabelle 2: Sanierungszustand der bis 1990 erbauten EZFH in der Stichprobe zu den Untersuchungsregionen (eigene Auswertung eines Datensatzes der co2online $\mathrm{GmbH}$ von 2014)

\section{Rahmenbedingungen für Sanierungsentscheidungen von Eigenheimbesitzer/innen}

Nachdem gezeigt wurde, dass trotz unterschiedlicher regionalökonomischer Situation die energetischen Sanierungsaktivitäten in den beiden Untersuchungsregionen recht ähnlich sind, soll dieser Abschnitt die Frage klären, inwiefern Gebäudeeigentümer/innen die wirtschaftliche und soziodemografische Situation ihrer Region wahrnehmen und diese in ihre Sanierungsentscheidung einbeziehen. Dieser Frage wurde in qualitativen Interviews mit Eigentümer/innen von EZFH in den Untersuchungsregionen nachgegangen (Gossen/Nischan 2014). Insgesamt wurden zwischen Mai und Juli 201460 leitfadengestützte persönliche beziehungsweise telefonische Interviews - je 30 pro Untersuchungsregion - geführt. Im Fokus der qualitativen Befragung standen mögliche Hemmnisse und Treiber, die einen Einfluss auf die Sanierungsentscheidung von privaten Eigentümer/innen haben. Ferner bezog sich das Erkenntnisinteresse auf die Nutzungsperspektiven der Hausbesitzer/innen und die Frage, ob ein Zusammenhang zwischen Nutzungsabsicht, Sanierungsentscheidung und der jeweiligen regionalökonomischen Situation besteht.

Der Großteil der Befragten nannte als Anlass für die Auseinandersetzung mit einer energetischen Sanierung notwendige Instandsetzungen aufgrund von Mängeln und ohnehin geplante Sanierungs-, Instandhaltungs- oder Modernisierungsmaßnahmen. Die wichtigsten Motive zur energetischen Sanierung umfassen Aspekte, die zu einer Aufwertung des Hauses während der eigenen Nutzung beitragen. So ist die Möglichkeit, Energiekosten infolge der Maßnahme einzusparen, der am häufigsten genannte Grund. Nahezu gleichrangig ist der Wunsch, die Wohnqualität zu verbessern, indem beispielsweise notwendige Reparaturen oder optische Verschönerungen erfolgen oder das Raumklima verbessert wird. Hausbesitzer/ innen, denen eine Unabhängigkeit von fossilen Energieträgern wichtig ist, denken über eine Umstellung ihrer Wärmeversorgung auf Erneuerbare Energien nach. Klimaschutz als Motiv spielt für diese Gruppe eine größere Rolle als für Befragte, die Sanierungsmaßnahmen an der Gebäudehülle vornehmen und beispielsweise das Dach oder die Fassade dämmen oder die Fenster austauschen.

Für die Eigentümer/innen ist es wichtig, dass sich die energetische Sanierung im Rahmen der eigenen Nutzungsdauer rechnet. Die Sanierungsmaßnahmen werden unter anderem danach ausgewählt, ob sie rentabel sind; gezielte Wirtschaftlichkeitsrechnungen werden dennoch nur selten aufgestellt. So sind auch die Möglichkeiten einer fachmännischen Absicherung, beispielsweise durch eine professionelle Energieberatung, zumeist unbekannt. Einsparungen von Energie und Kosten werden also erwartet, aber im Voraus nicht quantifiziert und auch nach der Sanierung nur selten systematisch erfasst. Trotzdem sehen die meisten Befragten rückblickend ihre Erwartungen an die Wirtschaftlichkeit der Sanierung erfüllt. 
Unter den Hemmnissen dominieren finanzielle Restriktionen. Entweder hält die mangelnde eigene Liquidität oder der fehlende Zugang zu Investitionskapital die Befragten von der Umsetzung einer Sanierung ab. Bautechnische Gebäudemerkmale, wie etwa Sichtmauerwerk oder Denkmalschutzauflagen spielen schon in einem frühen Stadium der Entscheidungsfindung eine wichtige Rolle und können insbesondere aufwendigen Maßnahmen entgegenstehen. Ferner beeinflussen einseitige Berichte in den Medien, beispielsweise über Probleme wie Schimmelbefall, und Erfahrungsberichte aus dem persönlichen Umfeld die allgemeinen Einstellungen zu energetischen Sanierungen und verfestigen Vorbehalte gegenüber bestimmter Maßnahmen. Dies ist besonders gravierend, wenn keine ergänzende Fachmeinung eingeholt wird. Ferner können ein Informationsdefizit beziehungsweise -überfluss als auch ein mangelhaftes Angebot an fachmännischen Handwerksunternehmen Sanierungen verzögern oder sogar verhindern.

Die qualitativen Ergebnisse zeigen also zunächst, dass Gebäudeeigentümer/innen vor allem individuelle Aspekte und Faktoren bei den Sanierungsanlässen, -motiven und -hemmnissen nennen, die nicht in direkter Abhängigkeit zu regionalökonomischen Faktoren stehen.

Allerdings lassen die Antworten verschiedene Zusammenhänge zwischen regionalökonomischer Situation und der Sanierungsentscheidung vermuten. Zum einen kann die Höhe des Haushaltseinkommens die Liquidität und die Kreditwürdigkeit beeinflussen. Zum anderen spielt die Nutzungsperspektive eine wichtige Rolle, das heißt die Frage, wie lange das Gebäude selbst genutzt wird und was danach mit der Immobilie passiert. Die Nutzungsperspektive ist in den Untersuchungsregionen unterschiedlich ausgeprägt. Zwar herrschen sowohl in Potsdam/Potsdam-Mittelmark als auch in LausitzSpreewald mit Blick auf die kurz- und mittelfristigen Planungen vor allem Eigennutzungsabsichten vor; aber die langfristigen Nutzungsperspektiven unterscheiden sich in Abhängigkeit der regionalen Situation doch deutlich. So bewerten die befragten Eigentümer/innen aus Lausitz-Spreewald die zukünftigen Miet- oder Verkaufsperspektiven für ihr Eigentum als wenig aussichtsreich und sehen auch vielfach Probleme hinsichtlich der Vererbung ihres Hauses an Nachkommen. Über die eigene Nutzungszeit hinaus werden keine langfristigen und strategischen Sanierungspläne verfolgt, da angenommen wird, dass der allgemeine Wertverlust von Immobilien in der Region auch durch eine energetische Sanierung nicht ausgeglichen werden kann. Demgegenüber wird in Potsdam/Potsdam-Mittelmark mit der energetischen Sanierung eine langfristige Wertsteigerung der Immobilie beabsichtigt.

Die regionalökonomische Situation kann zudem die Sanierungsdurchführung beeinflussen. Energetisch saniert wird zwar in beiden Regionen, jedoch in unterschiedlicher Tiefe und Qualität. In beiden Regionen überwiegen die professionell durchgeführten Sanierungsarbeiten, wobei in Lausitz-Spreewald recht viele Sanierungsschritte in Eigenleistung erbracht werden - meistens handelt es sich um Vor- oder Nachbereitun- gen der anspruchsvolleren und von Unternehmen ausgeführten Arbeiten. Außerdem laufen in Lausitz-Spreewald die Sanierungen in Abhängigkeit von den zur Verfügung stehenden geringeren finanziellen Mitteln vielfach eher schrittweise ab.

\section{Schlussfolgerungen}

Der vorliegende Beitrag untersucht, inwiefern die regionalökonomische Situation einen Einfluss auf die individuelle energetische Sanierungsentscheidung und somit auf den energetischen Zustand von EZFH hat. Obwohl beide Untersuchungsregionen deutliche Unterschiede im Hinblick auf die regionalökonomische Situation aufweisen, bestehen kaum Unterschiede hinsichtlich Art und Umfang der Sanierung, soweit dies auf der Basis der zur Verfügung stehenden Daten abgeschätzt werden konnte.

Erklärt werden kann diese Diskrepanz zunächst durch die eher geringe Bedeutung, die Eigentümer/innen regionalen Faktoren sowie Fragen der Nachnutzung der Immobilie geben. Gleichzeitig spielen jedoch finanzielle Hemmnisse eine wichtige Rolle, sodass die regionale Einkommenshöhe und Beschäftigungsmöglichkeiten indirekt durchaus von Bedeutung für Sanierungsmaßnahmen sind. Hinzu kommt, dass Nachwendeeffekte in den Untersuchungsregionen scheinbar andere Einflussfaktoren überlagern. So ist bekannt, dass es in den 1990er Jahren in den neuen Bundesländern im Vergleich zu den alten Bundesländern bei MFH höhere Sanierungsaktivitäten gab, die entsprechend zu geringeren Verbräuchen führten (Michelsen et al. 2014). Unsere Untersuchungen bestätigen, dass dies auch für EZFH in der ostdeutschen Untersuchungsregion gilt. Unterschiede zum bundesweiten Sanierungsverhalten bestehen auch noch in den 2000er Jahren, die sich teilweise als auf den Sanierungshöhepunkt folgende Flaute interpretieren lassen.

Regionale Unterschiede im Sanierungsverhalten wurden bislang nicht nur zwischen Ost- und Westdeutschland sondern auch zwischen Nord- und Süddeutschland festgestellt (Diefenbach et al. 2010). Wenn die regionalökonomische Situation hierfür von eher geringer Bedeutung ist, stellt sich die Frage, woher die regionalen Unterschiede resultieren. Ein Erklärungsansatz können Differenzen bei Beratungs- und Förderangeboten sein, denn diese weisen deutliche Unterschiede zwischen Bundesländern, Regionen und Kommunen auf. Auffällig ist, dass es in Ostdeutschland nur wenige regionale Beratungs- und Förderangebote für Wohneigentümer/innen gibt im Vergleich zu Westdeutschland wie beispielsweise Nordrhein-Westfalen oder Baden-Württemberg. Wie die qualitativen Befragungen in den Untersuchungsregionen zeigen, sind professionelle Energieberatungsangebote den Sanierer/innen meist unbekannt. Dies könnte - neben dem historisch bedingten Sanierungshöhepunkt der 1990er Jahre und der darauf folgenden Sanierungsflaute in den 2000ern - erklären, warum in den letzten Jahren die Sanierungsaktivitäten in Westdeutschland die in Ostdeutschland übertrafen. 
Aus Klimaschutzsicht ist weniger die Sanierungsrate als vielmehr die tatsächliche Reduktion des Energieverbrauchs also die Wirkung der Sanierung - relevant. Der Heizenergieverbrauch liegt dabei in den Untersuchungsregionen trotz eines höheren Anteils an vollsanierten Gebäuden nicht unter dem bundesweiten Durchschnitt. Dies deutet auf eine geringere Sanierungstiefe oder -qualität hin. Erklären lässt sich dies mit der überdurchschnittlichen Sanierungsaktivität in den 1990er Jahren, in denen das Sanierungsniveau noch deutlich unter dem Standard der 2000er Jahre lag.

Da Sanierungsmaßnahmen an der Gebäudehülle eine lange Lebensdauer aufweisen, wirken sich Versäumnisse bei Sanierungstiefe und -qualität langfristig aus und können dazu führen, dass ein klimaneutraler Gebäudebestand bis zum Jahr 2050 kaum erreicht werden kann. Insofern ist es zur Erreichung der klimapolitischen Ziele wichtig, dass sich Sanierungsrate und -tiefe in den Untersuchungsregionen steigern. Hierfür bedarf es weiterer Anstrengungen auch auf regionaler Ebene, um Beratungsangebote und Fördermöglichkeiten vor Ort zu schaffen und bekannt zu machen.

\section{Anmerkung}

[1] Das Projekt „Gebäude-Energiewende“ ist ein Verbundvorhaben von IÖW, BTU Cottbus-Senftenberg und RWTH Aachen University. Es wird durch das BMBF im Rahmen des Förderschwerpunkts Sozialökologische Forschung gefördert. Mehr Informationen unter www.gebaeude-energiewende.de.

\section{Literatur}

AStBB (Amt für Statistik Berlin-Brandenburg) (2011): Statistischer Bericht F I 1-j/10. Fortschreibung des Wohngebäude und Wohnungsbestandes im Land Brandenburg am 31. Dezember (2010). Internet: www.destatis.de (abgerufen 19.02. 2015).

AStBB (2012a): Bevölkerungsprognose für das Land Brandenburg (AI8-11). Internet: www.demografie.brandenburg.de (abgerufen am 20.09. 2013).

AStBB (2012 b): Statistisches Jahrbuch 2012 - Brandenburg. Internet: www.statistik-berlin-brandenburg.de (abgerufen 09. 01. 2014).

AStBB (2012 c): Statistischer Bericht - Ergebnisse des Mikrozensus im Land Brandenburg 2011 - Bevölkerung und Erwerbstätigkeit. Internet: www.statistik-berlin-brandenburg.de (abgerufen 15.01.2014).

AStBB (2014): Zensus 2011, für das Projekt bereitgestellte Datensätze. Potsdam.

BMWi (Bundesministerium für Wirtschaft und Energie) (2014a) Zahlen und Fakten Energiedaten. Internet: www.bmwi.de (abgerufen 22. 04. 2015).

BMWi (2014b): Zeitreihen zur Entwicklung der erneuerbaren Energien in Deutschland. unter Verwendung von Daten der Arbeitsgruppe Erneuerbare Energien-Statistik (AGEE-Stat) (Stand August 2014). Internet: www.erneuerbare-energien.de (abgerufen 09.01. 2015).

BMWi und BMU (Bundesministerium für Umwelt, Naturschutz und Reaktorsicherheit) (2010): Energiekonzept für eine umweltschonende, zuverlässige und bezahlbare Energieversorgung. Internet: www.bmu.de (abgerufen 26.01. 2011).

Brandenburgisches Landesamt für Denkmalpflege: DB1 Denkmaldatenbank. Internet: ns.gis-bldam-brandenburg.de/hida4web/ search?smode=advanced (abgerufen 08.07.2014).

Diefenbach, N. et al. (2010): Datenbasis Gebäudebestand. Datenerhebung zur energetischen Qualität und zu den Modernisierungstrends im deutschen Wohngebäudebestand. Darmstadt.

Dunkelberg, E./Weiß, J. (2015): Zustand der Wohngebäude in wachsenden und schrumpfenden Regionen. Gebäudeeigenschaften, Sanierungszustand und Energieverbrauch der Wohngebäude in den Regionen Lausitz-Spreewald und Potsdam/Potsdam-Mittelmark. Berlin.

Gossen, M./Nischan, C. (2014): Regionale Differenzen in der Wahrnehmung von energetischen Sanierungen. Ergebnisse einer qualitativen Befragung von privaten Gebäudeeigentümerlnnen zu energetischer Sanierung in zwei unterschiedlichen Regionen. Berlin.

Jahnke, K. (2013): co2online Gebäudedaten. Auswertungen und Nutzen für die Forschung und Praxis. Unveröffentlicht.

Jochum, P. et al. (2012): Technische Restriktionen bei der energetischen Modernisierung von Bestandsgebäuden. Endbericht. Berlin/Heidelberg.

Loga, T. et al. (2007). Energieeffizienz im Wohngebäudebestand - Techniken, Potenziale, Kosten und Wirtschaftlichkeit. Studie im Auftrag der Südwestdeutschen Wohnungswirtschaft e. V. Darmstadt.

Michelsen, C. et al. (2014): Wärmemonitor Deutschland 2013: Gesunkener Heizenergiebedarf, gestiegene Kosten. In: DIW Wochenbericht 41. S. 1015-1027.

DESTATIS (2014): Zensus 2011, für das Projekt bereitgestellte Datensätze. Wiesbaden.

DESTATIS (2012): Bevölkerung und Erwerbstätigkeit, Haushalte und Familien - Ergebnisse des Mikrozensus. Internet: www.destatis.de (abgerufen 13.01.2014).

Stieß, I. et al. (2010): Handlungsmotive, -hemmnisse und Zielgruppen für eine energetische Gebäudesanierung - Ergebnisse einer standardisierten Befragung. Frankfurt.

Weiß, J./Dunkelberg, E. (2010): Erschließbare Energieeinsparpotenziale im Ein- und Zweifamilienhausbestand. Berlin.

Zensus (2011): Zensusdatenbank Zensus 2011 der Statistischen Ämter des Bundes und der Länder. Individuelle Abfrage. Internet: ergebnisse. zensus2011.de/\#dynTable:statUnit=GEBAEUDE;absRel=ANZAHL;ags= 12; agsAxis=X;yAxis=ZAHLWOHNGN_HHG:sum:1:2,EIGENTUM:sum:2 (abgerufen12.02.2015).

AUTORINNEN + KONTAKT

Dr.Julika Weiß, Dr. Elisa Dunkelberg und Maike Gossen sind wissenschaftliche Mitarbeiterinnen am Institut für ökologische Wirtschaftsforschung (IÖW) in den Forschungsfeldern Nachhaltige Energiewirtschaft und Klimaschutz beziehungsweise Unternehmensführung und Konsum.

IÖW, Potsdamer Str. 105, 10785 Berlin, Tel.: +49 30 884594-0.

E-Mail: julika.weiss@ioew.de, elisa.dunkelberg@ioew.de,maike.gossen@ioew.de

Doreen Großmann ist wissenschaftliche Mitarbeiterin im Fachgebiet Management regionaler Energieversorgungsstrukturen an der Brandenburgischen Technischen Universität Cottbus-Senftenberg.

Brandenburgische Technische Universität CottbusSenftenberg, Großenhainer Str. 57, 01968 Senftenberg, Tel.: +49357385506. E-Mail: doreen.grossmann@b-tu.de
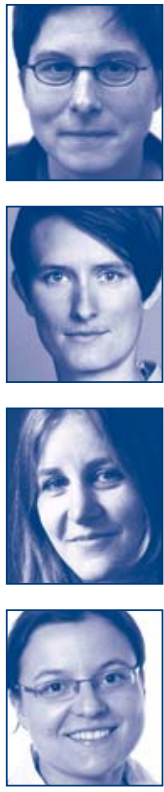